\title{
Thoughts on entropic gravity in the Parikh-Wilczek tunneling model of Hawking radiation
}

\author{
Wen-Yu Wen ${ }^{1,2, a}$ \\ ${ }^{1}$ Department of Physics and Center for High Energy Physics, Chung Yuan Christian University, Chung Li, Taiwan \\ ${ }^{2}$ Leung Center for Cosmology and Particle Astrophysics, National Taiwan University, Taipei 106, Taiwan
}

Received: 20 December 2013 / Accepted: 17 May 2014 / Published online: 4 June 2014

(C) The Author(s) 2014. This article is published with open access at Springerlink.com

\begin{abstract}
In this letter, we use the Parikh-Wilczek tunneling model of Hawking radiation to illustrate that a reformulation of Verlinde's entropic gravity is needed to derive Newton's law for a temperature-varying screen, required by the conservation of energy. Furthermore, the entropy stored in the holographic screen is shown to be additive and its temperature dependence can be obtained.
\end{abstract}

\section{Verlinde's entropic gravity and holographic screen}

To explain why gravity is so different from the other three forces in the nature, Verlinde proposed that gravity could be regarded as an entropic force caused by changes in the information associated with the positions of material bodies [1]. His idea can be illustrated by a particle with mass $m$ approaching some hypothesized holographic screen. The screen bounds the emerged part of space containing the particle, and it stores data that describe the part of space that has not yet emerged, as well as some part of the emerged space. The change in entropy on the screen, denoted $\Delta S$, is assumed to be linear in the particle's displacement $\Delta x$ as follows:

$\Delta S=2 \pi k_{B} \frac{m c}{\hbar} \Delta x$.

If one further assumes that the screen is thermalized at the Unruh temperature set up by particle's acceleration, then Newton's second law can be derived from the entropic force relation:

$F \Delta x=T \Delta S$.

One opposition to this proposal is given by [2] using the measurement result of quantum states of ultra-cold neutron under the Earth's gravity. According to Kobakhidze's argument, a pure state neutron would have evolved into a mixed

a e-mail: steve.wen@gmail.com state thanks to $\Delta S>0$ in (1). This criticism, however, is questioned in [3] and one resolution was suggested in [4] by abandoning the implicit assumption in [2] that the entropy on the holographic screen is additive. Instead, unitarity could still be restored even with $\Delta S>0$ if bits on the screen were entangled in some delicate way. Nevertheless, it remains unclear how the entropy is entangled on the holographic screen.

The other restriction of (2) is that a holographic screen at thermal equilibrium corresponds to the particle moves in uniform acceleration. A generalization of the entropic force relation is needed to describe a particle with generic motion. Similar to the generalization of Newton's second law to include a varying mass, it is natural to generalize the entropic gravity to include a varying screen temperature. Having said that, we consider an adiabatic process such that the temperature of the holographic screen is slowly varying but still well defined while a massive particle moves relatively to the screen. The generalized entropic force formula then becomes

$F \Delta r=\Delta(T S)$,

for varying screen temperature $T$ and screen entropy $S$, while a particle is displaced a distance $\Delta r$ from the screen at fixed location.

In the following, we will illustrate that Verlinde's screen could be realized in the tunneling model of Hawking radiation if a reformulation of entropic gravity is adopted to compensate for the effect of varying temperature. Following a similar argument as in [2] but for temperature-varying screen, we show that the entropy can still be additive on the screen and its temperature dependence can be derived.

\section{Entropic gravity with temperature-varying screen}

The original treatment of Hawking radiation by Hawking is to consider a perturbation in a fixed background of the 
Schwarzschild black hole. The thermal spectrum brought about controversial debates over the Information Loss Paradox. Parikh and Wilczek considered radiation as an outgoing tunneling particle where the conservation of energy is enforced [5]. It was later confirmed that in their model information is also conserved by computing the mutual information for two successive radiations [6]. If gravity has an entropic origin, it is desirable to describe the Hawking radiation (as a tunneling process), at least for Schwarzschild black holes, as some form of entropy change in a holographic screen. We recall that back reaction from radiated particle to the black hole makes it a system away from thermal equilibrium, namely with temperature varying or being not well defined. As a result it is proper to apply the general formula (3) rather than (2) to the tunneling model. For a screen which stores the same amount of information as the black hole entropy and possesses the same temperature as the black hole, the change on the right-hand side before and after radiation can be computed as

$$
\begin{aligned}
\Delta(T S)= & \frac{1}{8 \pi G(M-\omega)} \cdot 4 \pi G(M-\omega)^{2} \\
& -\frac{1}{8 \pi G M} \cdot 4 \pi G M^{2}=-\frac{\omega}{2},
\end{aligned}
$$

which can be understood as a quantum of mass (energy) $\omega$ that is discarded from the black hole up to a factor $1 / 2 .{ }^{1}$ The left-hand side computes the required work by pulling a pointlike varying mass (energy) from the black hole. According to Newton's law of gravity, that is

$$
\int_{2 G M}^{2 G(M-\omega)} F(r) \mathrm{d} r=-\left.\frac{G\left(M-\frac{r}{2 G}\right)\left(\frac{r}{2 G}\right)}{r}\right|_{2 G M} ^{2 G(M-\omega)}=-\frac{\omega}{2},
$$

which is consistent with the right-hand side. Therefore, the gravitational force can again be identified with an entropic force, even for a nonequilibrium system.

\section{Holographic screen in the Parikh-Wilczek tunneling model of Hawking radiation}

Inspired by the reformulation of entropic gravity in the previous discussion, we are motivated to study a holographic screen at arbitrary position but admitting a varying temperature, in reaction to the displacement of a radiation particle. For symmetry reasons, a spherical screen enclosing the black

\footnotetext{
1 This factor has been pointed out in the [7] as equipartition of energy in the horizon degrees of freedom. In brief, each quantum of area fluctuation contributes an energy $T / 2$, that is, $E=\frac{1}{2} n T$ for $n=A=4 S$. One can derive (4) for loss of energy (mass) $\omega$.
}
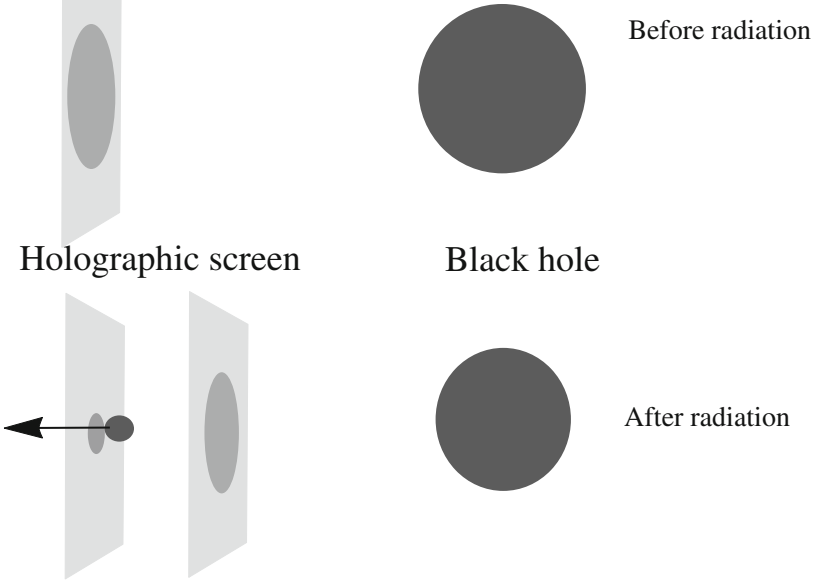

Fig. 1 A schematic drawing of a particle radiated out of a black hole and their holographic screen

hole seems to be a good choice. We denote the information stored on a holographic screen associated to a black hole with mass $M$ as $S_{M}(r)$. The screen is located at some distance $r$ from the center of the black hole. We may want to have $r>2 G M$ to avoid ambiguity of a screen inside the black hole. Before proceeding with the general formula (3), we would like to first point out some difficulties appearing in the original formulation of the entropic gravity (2) in the tunneling model of black hole radiation. If we assume that the entropy stored on the screen follows a simple coarse grained relation as shown in Fig. 1, following the neutron-earth system in [2]:

$S_{M}(r+\Delta r)=S_{\omega}(r+\Delta r)+S_{M-\omega}(r)$,

where $\Delta r$ is the distance between radiated mass and the screen. For an infinitesimal displacement, we have $S_{M}(r+$ $\Delta r) \simeq S_{M}(r)+\Delta S$. If the entropy on the screen were additive, that is,

$S_{M-\omega}(r)=S_{M}(r)-S_{\omega}(r)$,

one obtains

$\Delta S_{M} \simeq S_{\omega}(r+\Delta r)-S_{\omega}(r)$.

Since $\Delta S_{M} \propto \Delta r$ for its entropic origin, we come to the same conclusion: that the translation operator is no longer Hermitian and disobeying quantum mechanics. In [4], a generalized entropy formula by Tsallis [8] was called upon to restore the unitarity of translation, where an additional entangled term was essential. In our radiation-black hole system, it was known that the Parikh-Wilczek model predicted a non-thermal radiation spectrum and correlation between two emissions $\omega_{1}, \omega_{2}$ was computed as $8 \pi \omega_{1} \omega_{2}$, known as 
mutual information [6]. The entanglement is necessary for the conservation of entropy. Therefore, it is possible to correct the additivity condition (7) to include a term $S_{\text {ent }}>0$ encoding entanglement between black hole and radiation:

$S_{M-\omega}(r)=S_{M}(r)-S_{\omega}(r)-S_{\mathrm{ent}}(r)$,

such that entropy is conserved before and after radiation. ${ }^{2}$ Notice that the relative sign for the last term is different from the usual entanglement between two subsystems. This difference in fact makes the situation worse since now $\Delta S_{M} \simeq S_{\omega}(r+\Delta r)-S_{\omega}(r)-S_{\text {ent }}(r)$. For a translation to be unitary, say $S_{\omega}(r+\Delta r)=S_{\omega}(r)$, one has $\Delta S_{M}<0$, implying a violation of the second law of thermodynamics, which reflects the fact that the black hole cannot evaporate classically. If the Hawking radiation were thermal, one might expect the increased entropy during thermal process to be large enough to compensate for the reduced entropy due to splitting of the radiated particle from the black hole. However, in Parikh-Wilczek's tunneling model, one has no such additional source for the entropy since the process is nonthermal.

Now we turn to an application of the general entropic formula (3). Because the temperature may vary for screens at different locations, we assume that the coarse grained relation (6) is modified as

$$
\begin{aligned}
T(r+\Delta r) S_{M}(r+\Delta r)= & T(r+\Delta r) S_{\omega}(r+\Delta r) \\
& +T(r) S_{M-\omega}(r) .
\end{aligned}
$$

If we assume a linear relation between screen temperature and small displacement, that is, $T(r+\Delta r) \simeq T(r)+\Delta T$, one obtains

$\Delta S_{M} \simeq\left[S_{\omega}(r+\Delta r)-S_{\omega}(r)\right]+\left[\frac{\Delta T}{T} S_{\omega}(r+\Delta r)-S_{\mathrm{ent}}\right]$.

For the translation of the radiated particle being unitary, $S_{\omega}(r)=S_{\omega}$ is a constant. Vanishing of $\Delta S_{M}$ for $\Delta r=0$ suggests $S_{\text {ent }}(r)=0$. According to (9), the entropy on the screen is additive. The temperature dependence can also be obtained by integration:

$S_{M}(r)-S_{M}\left(r_{0}\right)=S_{\omega} \ln \frac{T(r)}{T\left(r_{0}\right)}$

for some reference position $r_{0}$. The movement of the radiated particle with respect to the screen does not change corre-

\footnotetext{
2 Classically, a black hole is forbidden to split because $S_{M}(r)>$ $S_{M-\omega}(r)+S_{\omega}(r)$. In the Parikh-Wilczek model, the entanglement provides an additional source of entropy to conserve the entropy during the evaporation process.
}

sponding to the entropy $S_{\omega}$. Instead, it feels different temperatures from screens at different locations. If one recalls the scenario of a massive particle tunneling out of black hole horizon: the radiated particle experiences less gravitational pull while it moves away from the black hole, as if it feels colder from holographic screen. The screen temperature reflects the acceleration or deceleration as what happens in the Unruh effect.

\section{Discussion}

In this letter, with the explicit example of Parikh-Wilczek's tunneling model of Hawking radiation, we illustrate that Verlinde's holographic screen could have violated unitarity of quantum mechanics if it were additive in entropy. This sickness cannot be simply cured by the assumption that the entropy on screen is entangled. Motivated by a general formula of entropic gravity, one shows that the entropy on the screen can still be additive if the relation between screen entropy and temperature is given by (12). Some comments are in order.

Firstly, the Parikh-Wilczek is a semiclassical model, which could receive a further quantum correction. For instance, a one-loop correction to the surface gravity was considered in [9]. As a result, the Bekenstein-Hawking area law receives a logarithmic correction, denoted $S_{\alpha}(M)=$ $4 \pi M^{2}-4 \pi \alpha \ln \left(1+\frac{M^{2}}{\alpha}\right)$, and the Hawking temperature is modified as $T_{H}=\frac{M^{2}+\alpha}{8 \pi M^{3}}$ for a large black hole, where the coefficient $\alpha$ is related to the trace anomaly. One can show that the relation of the generalized entropic force (3) is no longer satisfied, since

$\Delta\left(T_{H} S_{\alpha}\right)=-\frac{\omega}{2}+\alpha\left[\frac{3}{2 M^{2}}-\frac{3 \alpha+M^{2}}{2 M^{4}} \ln \left(1+\frac{M^{2}}{\alpha}\right)\right] \omega$.

Nevertheless, this might be fixed from two different viewpoints. One way is to modify Newton's gravitational force by a quantum correction up to $\mathcal{O}\left(\alpha^{2}\right)$, such that $\int F_{\alpha}(r) \mathrm{d} r$ agrees with the above result. The other way is to rederive the Hawking temperature, instead of that obtained in the small $\omega$ limit. The proper definition of the Hawking temperature $T_{\alpha}(M)$ is given by

$T_{\alpha}(M-\omega) S_{\alpha}(M-\omega)-T_{\alpha}(M) S_{\alpha}(M)=-\frac{\omega}{2}$.

It seems unlikely to have analytic forms for loop corrected $F_{\alpha}(r)$ and $T_{\alpha}(M)$, but at least in principle one can get the order expansion for small $\alpha$. Once the relation (3) can be fixed, our conclusion for the temperature-varying screen will still be valid, at least perturbatively in terms of $\alpha$. 
Secondly, although Verlinde's adoption of entropic force was meant to tame the wild gravity beast, it is natural to ask how to incorporate the other forces in his entropic formalism. This generalization has been considered for the electromagnetic force, by inclusion of the chemical potential in the first law of thermodynamics [10]. In particular, for the tunneling model of Reissner-Nordström black hole radiation [11], one expects that the relation (3) is generalized as

$F \Delta r=\Delta(T S-A Q)$,

where the gauge potential and total black hole charges are identified as the chemical potential and number density on the screen, respectively. On the left-hand side, one has to include the electromagnetic force for a consistent result. If there exists a holographic screen for charged black holes, it must also carry degrees of freedom for charges. The black hole censorship constraint $Q \leq M$ would translate into an upper bound for the charge density on the screen. The break down of the screen for excess charge would correspond to a possible naked singularity or closed time-like curve. The recently found charge-mass ratio bound for emission from the RN black hole [12] seems to support this picture by stating that a part of screen, which corresponds to the radiated particle, also has an upper bound for the charge density.

Acknowledgments WYW is supported in part by the Taiwan's National Science Council under Grant NO. 102-2112-M-033-003-MY4 and the National Center for Theoretical Science.

Open Access This article is distributed under the terms of the Creative Commons Attribution License which permits any use, distribution, and reproduction in any medium, provided the original author(s) and the source are credited.

Funded by SCOAP $^{3}$ / License Version CC BY 4.0.

\section{References}

1. E.P. Verlinde, On the origin of gravity and the laws of Newton. JHEP 1104, 029 (2011)

2. A. Kobakhidze, Gravity is not an entropic force. Phys. Rev. D 83, 021502 (2011)

3. M. Chaichian, M. Oksanen, A. Tureanu, On gravity as an entropic force. Phys. Lett. B 702, 419 (2011)

4. E.M.C. Abreu, J.A. Neto, Considerations on gravity as an entropic force and entangled states. Phys. Lett. B 727, 524 (2013)

5. M.K. Parikh, F. Wilczek, Hawking radiation as tunneling. Phys. Rev. Lett. 85, 5042 (2000)

6. B. Zhang, Q.-Y. Cai, L. You, M.-S. Zhan, Hidden messenger revealed in Hawking radiation: a resolution to the paradox of black hole information loss. Phys. Lett. B 675, 98 (2009)

7. T. Padmanabhan, Equipartition of energy in the horizon degrees of freedom and the emergence of gravity. Mod. Phys. Lett. A 25, 1129 (2010)

8. C. Tsallis, Possible generalization of Boltzmann-Gibbs statistics. J. Stat. Phys. 52(1-2), 479-487 (1988)

9. R. Banerjee, B.R. Majhi, Quantum tunneling and back reaction. Phys. Lett. B 662, 62 (2008)

10. Y.-X. Chen, J.-L. Li, First law of thermodynamics on holographic screens in entropic force frame. Phys. Lett. B 700, 380 (2011)

11. J.-Y. Zhang, Z. Zhao, Hawking radiation of charged particles via tunneling from the Reissner-Nordström black hole. JHEP 0510, 055 (2005)

12. K.K. Kim, W.-Y. Wen, Charge-mass ratio bound and optimization in the Parikh-Wilczek tunneling model of Hawking radiation. Phys. Lett. B 731, 307 (2014) 